$\sum_{\text {Matthew N. Hurley }}$

(1]

Paediatric Respiratory Medicine, Nottingham Children's Hospital, Nottingham, UK.
(2)

matthew.hurley@nuh.nhs.uk

Y

@matthewnhurley

\title{
Staphylococcus aureus in cystic fibrosis: problem bug or an innocent bystander?
}

\section{A common organism}

Staphylococcus aureus is a commonly encountered organism in day-to-day living. However, the epidemiology is complicated by three different patterns of carriage. Up to $60 \%$ of the population hosts the organism at any one time and, while $\sim 20 \%$ are considered "persistent carriers" due to their status of being continuous hosts of the same strain, a further $20 \%$ never host S. aureus and so are considered non-carriers [1]. S. aureus is commonly encountered in childhood with nasopharyngeal carriage among healthy children as high as $48 \%$ in the USA [2] and $36 \%$ in the Netherlands [3]. S. aureus carriage varies markedly by occupation, as do the proportions of those who carry antibiotic resistant strains (methicillin-resistant S. aureus (MRSA)) [1].

\section{Epidemiology}

In children with cystic fibrosis (CF) infection rates appear to vary considerably over time. The direction of this change appears to be locality specific: $S$. aureus infections have risen dramatically in the USA over time from $30 \%$ in 1990 to $60 \%$ in 2016 [4]. This is supported by a randomised trial in the USA from 1987 to 1989 , reporting $30.4 \%$ of infants in the placebo group had S. aureus isolated from the respiratory tract [5]. Data for the UK over the same time period is less easily obtained, but appears to show the opposite trend. In 1994 WeAVER et al. [6] reported a randomised trial of anti-staphylococcal antibiotic prophylaxis in 38 babies where $60 \%$ of babies in the nonprophylaxis group had $S$. aureus cultured from their respiratory tract. The Cochrane review [7] reports data from the Chatfield study (1991) in which 37\% of children in the placebo group cultured S. aureus. Current data from the UK CF Registry suggest that the proportions of children in whom S. aureus is detected is reducing, with $16 \%$ of $0-3$ year-olds and 23.7 of 4-7 year-olds documented as having intermittent $S$. aureus infection in the UK [8].

Infection rates also vary considerably by country. While comparisons of infection rates between countries is wrought with methodological difficulties, significant differences between the UK and USA have been reported in terms of age at first infection [9], and a three-fold greater annual prevalence of methicillin-sensitive $S$. aureus and an eight-fold greater annual prevalence of MRSA in US CF centres compared to those in the UK [10].

Rates throughout Europe appear equally varied; however, direct comparisons are complicated by the markedly different population size of patients and registry coverage rates of patients in each country. The European Cystic Fibrosis Society Patient Registry
Cite as: Hurley MN. Staphylococcus aureus in cystic fibrosis: problem bug or an innocent bystander? Breathe 2018; 14: 87-90. 
(2015) reports the proportion of children with chronic S. aureus infection by country. This varies from as low as $8 \%$ in the UK to $68 \%$ in Latvia [11]. In adults with CF, S. aureus infection rates appear to reduce with increasing age through adulthood $[4,8]$.

\section{Infection or respiratory commensal?}

While it is established that $S$. aureus is a relatively common organism isolated from patients with $\mathrm{CF}$, the role that $S$. aureus plays in influencing respiratory health is less clear. The main challenge in this regard is determining true infection from colonisation. As implied by the high carriage rates in the healthy population, not all isolation from the upper respiratory tract represents lower respiratory tract infection. The paucity of evidence supporting any particular approach for managing $S$. aureus infection in young children is acknowledged [12].

\section{Microbiology}

In vitro and animal model studies suggest that those with CF exhibit dysregulated inflammatory responses to $S$. aureus [13] and the organism may even survive within macrophages [14]. S. aureus is equally implicated in early lung damage in such studies [15] and detection is independently associated with lower respiratory tract inflammation [16].

Just as Pseudomonas aeruginosa may select a mode of growth to promote chronic infection, so may $S$. aureus by selecting for small colony variants (SCVs). SCVs are part of the regular growth cycle, but, under particular conditions, this phenotype may predominate and form a persistent, intracellular, infection in the host through intrinsic antibiotic resistance without evoking the host immune response [17].

There is also an increasing body of literature that describes the complexities of co-infection with S. aureus and P. aeruginosa. Unfortunately, much of this is contradictory. There is evidence to suggest that, within the competitive niche of the CF lung, $P$. aeruginosa may force $S$. aureus to use a suboptimal metabolism strategy that eventually renders S. aureus unviable [18]. However, others have shown similar competitive strategies exhibited by $P$. aeruginosa may actually confer a survival benefit to $S$. aureus, protecting it from the effects of commonly used aminoglycoside antibiotics [19].

\section{Clinical effects of infection}

Chronic infection with $S$. aureus is similarly difficult to understand. High bacterial density, frequent exacerbations, evidence of inflammation (elevated interleukin-6 levels), presence of S. aureus SCVs and co-infection with Stenotrophomonas maltophilia appear to be particular risk markers for more severe lung disease [20]. SCVs appear to be a particular risk for worse lung function in the paediatric age group [21]. Illustrating the complexity, however, in another series $S$. aureus infection in the absence of other infections appeared to be a marker for more mild disease [22]

\section{Can we prevent infection, and is there a cost?}

In terms of management, the first consideration is whether prevention of infection in young children is both possible and confers benefit. The Cochrane review, which considered four trials of which one was a double-blind randomised controlled trial, concluded that fewer children receiving prophylaxis had a positive isolate of $S$. aureus [7]. The clinical consequences of this remain unclear. The only double-blind randomised trial of antibiotic prophylaxis used cephalexin and observed a delay in detection of $S$. aureus, but an increase in detection of $P$. aeruginosa [5]. This competing tension has led to differing approaches internationally [23-25]. In the UK, anti-staphylococcal antibiotic prophylaxis in the form of flucloxacillin, is recommended for the first 3 years. In the USA, prophylaxis is recommended against.

An Australian observational study using bronchoalveolar lavage-based microbiological sampling found that co-amoxiclav (amoxillinclavulanate) antibiotic prophylaxis use was not associated with either detection of $P$. aeruginosa or S. aureus [26], although an excess of $P$. aeruginosa isolates was noted in the prophylaxis group. Continuous anti-staphylococcal prophylaxis was associated with increased isolation of $P$. aeruginosa in an analysis of German CF Registry data [27] and more recently flucloxacillin was associated with an increased risk of earlier age of first $P$. aeruginosa detection [9].

Calls for an adequately powered randomised controlled trial of anti-staphylococcal antibiotic prophylaxis have been made for at least 20 years [28]. Fortunately, the CF-START trial (www. nets.nihr.ac.uk/projects/hta/142223; https://doi. org/10.1186/ISRCTN18130649) is now underway and so an answer to this critical question should be available to inform practice in the future.

\section{MRSA}

MRSA may be of particular concern as this has been associated with an increased rate of decline in lung function (as measured by forced expiratory volume in $1 \mathrm{~s}$ ) [29] and an increased risk of death [30]. The potential for eradication of newly acquired MRSA infection has recently been demonstrated [31, 32], although the clinical sequelae of this has yet to be 
demonstrated. Unfortunately evidence to support eradication of chronic MRSA infection is currently lacking [33].

\section{A pragmatic approach, but more evidence is needed}

When confronted with a positive $S$. aureus isolate in a patient with CF, management decisions are difficult and so largely dependent on the individual circumstances and clinical condition.
The pragmatic approach to early infection in young children is to treat positive cultures as they present; acknowledging that in upper airway cultures the potential for treating an upper airway commensal is high. Equally, the approach to managing the first MRSA isolate should be to attempt eradication with an approach that appears to be effective [32].

The questions of what are the optimal approaches for prevention of early infection and how best to manage patients with chronic infection remain accompanied by considerable uncertainty. One comfort is that we should have the answer to at least one of these questions in the near future.

\section{Conflict of interest}

None declared.

\section{References}

1. Kluytmans J, van Belkum A, Verbrugh H. Nasal carriage of Staphylococcus aureus: epidemiology, underlying mechanisms, and associated risks. Clin Microbiol Rev 1997; 10: 505-520.

2. Rosenfeld M, Bernardo-Ocampo C, Emerson J, et al. Prevalence of cystic fibrosis pathogens in the oropharynx of healthy children and implications for cystic fibrosis care. J Cyst Fibros 2012; 11: 456-457

3. Bogaert D, van Belkum A, Sluijter M, et al. Colonisation by Streptococcus pneumoniae and Staphylococcus aureus in healthy children. Lancet 2004; 363: 1871-1872.

4. Cystic Fibrosis Foundation. Patient Registry: 2016 Annual Data Report. Bethesda, Cystic Fibrosis Foundation, 2017.

5. Stutman HR, Lieberman JM, Nussbaum E, et al. Antibiotic prophylaxis in infants and young children with cystic fibrosis: a randomized controlled trial. J Pediatr 2002; 140: 299-305.

6. Weaver LT, Green MR, Nicholson K, et al. Prognosis in cystic fibrosis treated with continuous flucloxacillin from the neonatal period. Arch Dis Child 1994; 70: 84-89.

7. Smyth AR, Rosenfeld M. Prophylactic anti-staphylococcal antibiotics for cystic fibrosis. Cochrane Database Syst Rev 2017 4: CD001912.

8. UK Cystic Fibrosis Trust. UK CF Registry. Annual Data Report 2016. London, Cystic Fibrosis Trust, 2017.

9. Hurley MN, Fogarty A, McKeever TM, et al. Early respiratory bacterial detection and anti-staphylococcal antibiotic prophylaxis in young children with cystic fibrosis. Ann Am Thorac Soc 2018; 15: 42-48.

10. Goss CH, Muhlebach MS. Review: Staphylococcus aureus and MRSA in cystic fibrosis. J Cyst Fibros 2011; 10: 298-306.

11.Zolin A, Orenti A, Naehrlich L, et al. ECFS Patient Registry annual data report - 2015 data. European Cystic Fibrosis Society Patient Registry, 2017.

12. Robinson KA, Saldanha IJ, McKoy NA. Management of infants with cystic fibrosis: a summary of the evidence for the cystic fibrosis foundation working group on care of infants with cystic fibrosis. J Pediatr 2009; 155: Suppl., S94-S105.

13. Bartlett JA, Ramachandran S, Wohlford-Lenane CL, et al. Newborn cystic fibrosis pigs have a blunted early response to an inflammatory stimulus. Am J Respir Crit Care Med 2016; 194: 845-854

14. Li C, Wu Y, Riehle A, et al. Staphylococcus aureus survives in cystic fibrosis macrophages, forming a reservoir for chronic pneumonia. Infect Immun 2017; 85: e00883-16.

15. Cigana C, Bianconi I, Baldan R, et al. Staphylococcus aureus impacts Pseudomonas aeruginosa chronic respiratory disease in murine models. J Infect Dis 2017; 217: 933-942.

16. Sagel S, Gibson R, Emerson J, et al. Impact of Pseudomonas and Staphylococcus infection on inflammation and clinical status in young children with cystic fibrosis. J Pediatr 2009; 154: 183-188.

17. Kahl BC. Small colony variants (SCVs) of Staphylococcus aureus - a bacterial survival strategy. Infect Genet Evol 2014; 21: 515-522

18. Filkins LM, Graber JA, Olson DG, et al. Coculture of Staphylococcus aureus with Pseudomonas aeruginosa drives $S$. aureus towards fermentative metabolism and reduced viability in a cystic fibrosis model. J Bacteriol 2015; 197: 2252-2264.

19. Hoffman LR, Deziel E, D’Argenio DA, et al. Selection for Staphylococcus aureus small-colony variants due to growth in the presence of Pseudomonas aeruginosa. Proc Natl Acad Sci USA 2006; 103: 19890-19895.

20. Junge S, Gorlich D, den Reijer $M$, et al. Factors associated with worse lung function in cystic fibrosis patients with persistent Staphylococcus aureus. PLoS One 2016; 11: e0166220.

21. Wolter DJ, Emerson JC, MCNamara S, et al. Staphylococcus aureus small-colony variants are independently associated with worse lung disease in children with cystic fibrosis. Clin Infect Dis 2013; 57: 384-391.

22. Ahlgren HG, Benedetti A, Landry JS, et al. Clinical outcomes associated with Staphylococcus aureus and Pseudomonas aeruginosa airway infections in adult cystic fibrosis patients. BMC Pulm Med 2015; 15: 67.

23. Smyth AR, Bell SC, Bojcin S, et al. European Cystic Fibrosis Society standards of care: best practice guidelines. J Cyst Fibros 2014; 13: S23-S42.

24. Cystic Fibrosis Trust. Standards for the Clinical Care of Children and Adults with Cystic Fibrosis in the UK. 2nd Edn. London, Cystic Fibrosis Trust, 2011.

25. Flume PA, O'Sullivan BP, Robinson KA, et al. Cystic fibrosis pulmonary guidelines: chronic medications for maintenance of lung health. Am J Respir Crit Care Med 2007; 176: 957-969.

26. Douglas TA, Brennan S, Gard S, et al. Acquisition and eradication of $P$. aeruginosa in young children with cystic fibrosis. Eur Respir J 2009; 33: 305-311.

27. Ratjen F, Comes G, Paul K, et al. Effect of continuous antistaphylococcal therapy on the rate of $P$. aeruginosa acquisition in patients with cystic fibrosis. Pediatr Pulmonol 2001; 31: 13-16.

28. Elborn JS. Treatment of Staphylococcus aureus in cystic fibrosis. Thorax 1999; 54: 377-378.

29. Dasenbrook EC, Merlo CA, Diener-West M, et al. Persistent methicillin-resistant Staphylococcus aureus and rate of FEV 1 decline in cystic fibrosis. Am J Respir Crit Care Med 2008; 178: 814-821. 
S. aureus in CF: problem bug or an innocent bystander?

30. Dasenbrook EC, Checkley W, Merlo CA, et al Association between respiratory tract methicillin-resistant Staphylococcus aureus and survival in cystic fibrosis. JAMA 2010; 303: 2386-2392.

31. Vallieres E, Rendall JC, Moore JE, et al. MRSA eradication of newly acquired lower respiratory tract infection in cystic fibrosis. ERJ Open Res 2016; 2: 00064-2015.
32. Muhlebach MS, Beckett V, Popowitch E, et al. Microbiological efficacy of early MRSA treatment in cystic fibrosis in a randomised controlled trial. Thorax 2017; 72: 318-326.

33. Ahmed MI, Mukherjee S. Treatment for chronic methicillin-sensitive Staphylococcus aureus pulmonary infection in people with cystic fibrosis. Cochrane Database Syst Rev 2016; 3: CD011581. 